\title{
SOME NONOSCILLATION CRITERIA FOR INCLUSIONS
}

\author{
RAVI P. AGARWAL ${ }^{\infty}$, SAID R. GRACE and DONAL O'REGAN
}

(Received 21 January 2002; revised 28 January 2004)

Communicated by $\mathrm{K}$. Wysocki

\begin{abstract}
New nonoscillatory criteria are presented for second order differential inclusions. The theory relies on Ky Fan's fixed point theorem for upper semicontinuous multifunctions.

2000 Mathematics subject classification: primary $34 \mathrm{~A} 60$.
\end{abstract}

\section{Introduction}

In [1] we initiated the study of nonoscillatory solutions to the differential inclusion

$$
\left(a(t) y^{\prime}(t)\right)^{\prime} \in F(t, y(t)) .
$$

Recall a nontrivial solution of (1.1) is called oscillatory if it has arbitrarily large zeros, otherwise it is called nonoscillatory. In the single valued case many nonoscillatory results are available in the literature; see [6-10] and the references therein. In this paper, by looking at the asymptotic behaviour at infinity, we are able to establish new nonoscillatory criteria for (1.1). We first discuss (1.1) when $\int^{\infty} d s / a(s)<\infty$ and we establish the existence of a nonoscillatory solution $y$ (here $y \geq 0$ ) to (1.1) with $\lim _{t \rightarrow \infty} y(t)=0$. Two results will be presented, one where $\lim _{t \rightarrow \infty} y(t) / \pi(t)>0$ and the other where $\lim _{t \rightarrow \infty} y(t) / \pi(t)=\infty$; here $\pi(t)=\int_{t}^{\infty} d s / a(s)$. The theory relies on Ky Fan's fixed point theorem in the Banach space setting. It is also possible (and we present this in Section 2) to discuss the case when $\int^{\infty} d s / a(s)$ is infinity if we use Ky Fan's fixed point theorem in the Fréchet setting.

Solutions to (1.1) will be sought in $B[T, \infty)$ and $C[T, \infty) ; T \geq 0$ will be suitably chosen. Recall $B[T, \infty)$ denotes the Banach space of all continuous, bounded real

(C) 2006 Australian Mathematical Society $1446-7887 / 06 \$ A 2.00+0.00$ 
valued functions on $[T, \infty)$ endowed with the usual supremum norm, that is, $|u|_{\infty}=$ $\sup _{t \in[T, \infty)}|u(t)|$ for $u \in B[T, \infty) . C[T, \infty)$ denotes the space of continuous real valued functions on $[T, \infty)$, the topology being that of uniform convergence on compact intervals on $[T, \infty)$.

We next stute Ky Fan's fixed point theorem [2] and we also state a compactness criterion [5] in $B[T, \infty)$.

THEOREM 1.1. Let $Q$ be a nonempty, closed, convex subset of a Fréchet space $E$ and $N: Q \rightarrow C K(Q)$ an upper semicontinuous, compact map; here $C K(Q)$ denotes the family of nonempty convex compact subsets of $Q$. Then there exists $x \in Q$ with $x \in N(x)$.

THEOREM 1.2. Let $E$ be an equicontinuous and uniformly bounded subset of the $B$ anach space $B[T, \infty)$. If $E$ is equiconvergent at $\infty$, it is also relatively compact.

\section{Differential inclusions}

In this section a variety of nonoscillation results will be presented for the differential inclusion

$$
\left(a(t) y^{\prime}(t)\right)^{\prime} \in F(t, y(t)), \quad t \geq t_{0} \geq 0,
$$

where the function $a$ is single valued and $F$ is a multifunction. Throughout this section the following conditions will be satisfied:

$$
a \in C\left(\left[t_{0}, \infty\right), \mathbb{R}^{+}\right)
$$

and

$$
\left\{\begin{array}{l}
F:\left[t_{0}, \infty\right) \times \mathbb{R} \rightarrow C K(\mathbb{R}) \text { is an } L^{1} \text {-Carathéodory multifunction: by } \\
\text { this we mean } \\
\text { (a) for each measurable } u:\left[t_{0}, \infty\right) \rightarrow \mathbb{R} \text {, the map } t \mapsto F(t, u(t)) \text { has } \\
\text { measurable single valued selections; } \\
\text { (b) for a.e. } t \in\left[t_{0}, \infty\right) \text {, the map } u \mapsto F(t, u) \text { is upper semicontinuous; } \\
\text { (c) for each } r>0, \text { there exists } h_{r} \in L^{1}\left[t_{0}, \infty\right) \text { with }|F(t, u)| \leq h_{r}(t) \\
\text { for a.e. } t \in\left[t_{0}, \infty\right) \text { and all } u \in \mathbb{R} \text { with }|u| \leq r \text {; here }|F(x, u)|= \\
\text { sup }\{|v|: v \in F(x, u)\} \text {. }
\end{array}\right.
$$

In [1] we initiated the study of nonoscillatory solution to (2.1), and we continue this study here. In particular, different types (that is, different asymptotic behaviour at infinity than that in [1]) of nonoscillatory solutions are discussed. In our first two results we will assume $\int_{t_{0}}^{\infty} d s / a(s)<\infty$. 
THEOREM 2.1. Suppose (2.2) and (2.3) hold and in addition assume the following five conditions are satisfied

$$
\begin{aligned}
& F:\left[t_{0}, \infty\right) \times(0, \infty) \rightarrow C K((-\infty, 0]) \\
& \pi\left(t_{0}\right)<\infty \text { where } \pi(t)=\int_{t}^{\infty} \frac{d s}{a(s)}
\end{aligned}
$$

$$
\left\{\begin{array}{l}
\text { there exists a single valued } L^{1} \text {-Carathéodory function } \\
G:\left[t_{0}, \infty\right) \times \mathbb{R} \rightarrow[0, \infty) \text { with }|F(u, z)| \leq G(u, z) \\
\text { for }(u, z) \in\left[t_{0}, \infty\right) \times(0, \infty)
\end{array}\right.
$$

$$
\exists \lambda>0 \text { with } \int_{t_{0}}^{\infty} G(s, \lambda \pi(s)) d s<\infty
$$

and

$$
\left\{\begin{array}{l}
\text { there exists a positive constant } M \text { such that if } \\
0<x \leq y \leq \lambda \pi\left(t_{0}\right) \text {, then } G(t, x) \leq M G(t, y) \\
\text { for } t \geq t_{0} .
\end{array}\right.
$$

Then there is a nonoscillatory solution y of $\left(a(t) y^{\prime}(t)\right)^{\prime} \in F(t, y(t))$, a.e. $t \geq T$, with $\lim _{t \rightarrow \infty} y(t)=0$ and $y(t) / \pi(t) \in[\lambda / 2, \lambda]$ for $t \geq T$; here $T$ is chosen as in (2.9). We also have

$$
\lim _{t \rightarrow \infty} \frac{y(t)}{\pi(t)}=c_{0} \in\left[\frac{\lambda}{2}, \lambda\right]
$$

REMARK 2.1. If $G$ is nondecreasing in the second variable (that is, if $0<x \leq y$ implies $G(t, x) \leq G(t, y)$ for $\left.t \geq t_{0}\right)$ then clearly (2.8) holds with $M=1$.

ProOF. From (2.7) there exists $T \geq t_{0}$ with

$$
\int_{T}^{\infty} G(s, \lambda \pi(s)) d s \leq \frac{\lambda}{2 M} .
$$

We wish to apply Theorem 1.1 with $E=\left(B[T, \infty),|\cdot|_{\infty}\right)$ and

$$
Q=\{y \in B[T, \infty): \lambda \pi(t) / 2 \leq y(t) \leq \lambda \pi(t) \text { for } t \geq T\} .
$$

Define a mapping $N: Q \rightarrow \mathscr{P}(E)$ (the power set of $E$ ) by (here $y \in Q$ ),

$$
N(y)(t)=\frac{\lambda}{2} \int_{t}^{\infty} \frac{d s}{a(s)}-\int_{t}^{\infty} \frac{1}{a(s)} \int_{T}^{s} F(u, y(u)) d u d s \quad \text { for } t \geq T .
$$

Note [4, Proposition 1.1, page 777] guarantees that $N: Q \rightarrow C(E)$; here $C(E)$ denotes the family of nonempty, convex subsets of $E$. We first show that

$$
N: Q \rightarrow C(Q) .
$$


For notational purposes for any $y \in Q$, let

$$
\mathscr{F}(y)=\left\{u \in L^{1}[T, \infty): u(t) \in F(t, y(t)) \text { for a.e. } t \in[T, \infty)\right\} .
$$

Let $y \in Q$, and take $w \in N(y)$. Then there exists $\tau \in \mathscr{F}(y)$ with

$$
w(t)=\frac{\lambda}{2} \int_{t}^{\infty} \frac{d s}{a(s)}-\int_{t}^{\infty} \frac{1}{a(s)} \int_{T}^{s} \tau(u) d u d s \quad \text { for } t \geq T .
$$

From (2.4) we have immediately that

$$
w(t) \geq \frac{\lambda}{2} \int_{t}^{\infty} \frac{d s}{a(s)}=\frac{\lambda}{2} \pi(t) \text { for } t \geq T .
$$

Also (2.6), (2.8), (2.9) and $y \in Q$ implies

$$
\begin{aligned}
w(t) & \leq \frac{\lambda}{2} \pi(t)+\int_{t}^{\infty} \frac{1}{a(s)} \int_{T}^{s} G(u, y(u)) d u d s \\
& \leq \frac{\lambda}{2} \pi(t)+M \int_{t}^{\infty} \frac{1}{a(s)} \int_{T}^{\infty} G(u, \lambda \pi(u)) d u d s \\
& \leq \frac{\lambda}{2} \pi(t)+\frac{\lambda}{2} \int_{t}^{\infty} \frac{d s}{a(s)}=\lambda \pi(t) \quad \text { for } t \geq T .
\end{aligned}
$$

As a result $\lambda \pi(t) / 2 \leq w(t) \leq \lambda \pi(t)$ for $t \geq T$ for each $w \in N(y)$. Thus (2.10) holds. Next we show

$$
N: Q \rightarrow C(Q) \text { is a compact map. }
$$

To see this we will use Theorem 1.2. Take any $y \in Q$ and $w \in N(y)$. Then there exists $\tau \in \mathscr{F}(y)$ with

Thus

$$
w(t)=\frac{\lambda}{2} \int_{t}^{\infty} \frac{d s}{a(s)}-\int_{t}^{\infty} \frac{1}{a(s)} \int_{T}^{s} \tau(u) d u d s \text { for } t \geq T .
$$

$$
|w(t)| \leq \frac{\lambda}{2} \pi(t)+M \int_{t}^{\infty} \frac{1}{a(s)} \int_{T}^{s} G(u, \lambda \pi(u)) d u d s \leq \lambda \pi(t)
$$

for $t \geq T$, and so for each $w \in N(y)$ we have $|w|_{\infty} \leq \lambda \pi\left(t_{0}\right)$. Thus the set

$$
Y=\{N y: y \in Q\} \text { is a uniformly bounded subset of } B[T, \infty) \text {. }
$$

Since for each $t \geq T$ we have $|w(t)| \leq \lambda \pi(t)$ for $w \in N(y)$, then the set $Y$ is equiconvergent at $\infty$. Next if $t_{1}, t_{2} \in[T, \infty)$ with $t_{1} \leq t_{2}$ we have

$$
\begin{aligned}
\left|w\left(t_{2}\right)-w\left(t_{1}\right)\right| & \leq \frac{\lambda}{2} \int_{t_{1}}^{t_{2}} \frac{d s}{a(s)}+M \int_{t_{1}}^{t_{2}} \frac{1}{a(s)} \int_{T}^{s} G(u, \lambda \pi(u)) d u d s \\
& \leq \lambda \int_{t_{1}}^{t_{2}} \frac{d s}{a(s)}
\end{aligned}
$$


for $w \in N(y)$. Now Theorem 1.2 guarantees that $Y$ is relatively compact in $B[T, \infty)$, and as a result (2.11) holds. Essentially the same reasoning as in [1] implies that

$$
N: Q \rightarrow C K(Q) \text { is an upper semicontinuous map. }
$$

Theorem 1.1 guarantees that there exists $y \in Q$ with $y \in N(y)$. That is for every $t \geq T$, we have

$$
y(t) \in \frac{\lambda}{2} \pi(t)-\int_{t}^{\infty} \frac{1}{a(s)} \int_{T}^{s} F(u, y(u)) d u d s
$$

Also since $y \in Q$ we have $y(t) / \pi(t) \in[\lambda / 2, \lambda]$ for $t \geq T$, and $\lim _{t \rightarrow \infty} y(t)=0$ since (2.5) implies

$$
\begin{aligned}
|y(t)| & \leq \frac{\lambda}{2} \pi(t)+M \int_{t}^{\infty} \frac{1}{a(s)} \int_{T}^{s} G(u, \lambda \pi(u)) d u d s \\
& \leq \lambda \pi(t) \rightarrow 0 \text { as } t \rightarrow \infty
\end{aligned}
$$

Now there exists $\tau \in \mathscr{F}(y)$ with

so

$$
y(t)=\frac{\lambda}{2} \pi(t)+\int_{t}^{\infty} \frac{1}{a(s)} \int_{T}^{s}[-\tau(u)] d u d s \text { for } t \geq T,
$$

$$
\begin{aligned}
\lim _{t \rightarrow \infty} \frac{y(t)}{\pi(t)} & =\frac{\lambda}{2}+\lim _{t \rightarrow \infty} \frac{\int_{t}^{\infty}\left(\int_{T}^{s}[-\tau(u)] d u\right) d s / a(s)}{\int_{t}^{\infty} d s / a(s)} \\
& =\frac{\lambda}{2}+\int_{T}^{\infty}[-\tau(x)] d x .
\end{aligned}
$$

REMARK 2.2. Minor adjustments in the analysis are needed if (2.4) is replaced by

$$
F:\left[t_{0}, \infty\right) \times(0, \infty) \rightarrow C K([0, \infty))
$$

We leave the details to the reader.

THEOREM 2.2. Suppose (2.2)-(2.6) hold and in addition assume the following conditions are satisfied

$$
\exists \lambda>0 \text { with } \int_{t_{0}}^{\infty} \frac{1}{a(s)} \int_{t_{0}}^{s} G(u, \lambda) d u d s<\infty
$$

and

$$
\left\{\begin{array}{l}
\text { there exists a positive constant } M \text { such that if } 0<x \leq \lambda, \\
\text { then } G(t, x) \leq M G(t, \lambda) \text { for } t \geq t_{0}
\end{array}\right.
$$


Then there is a nonoscillatory solution y of $\left(a(t) y^{\prime}(t)\right)^{\prime} \in F(t, y(t))$, a.e. $t \geq T$ with $\lim _{t \rightarrow \infty} y(t)=0$ and $y(t) \leq \lambda$ for $t \geq T$; here $T$ is chosen as in (2.20). If in addition

$$
\left\{\begin{array}{l}
\text { there exists a single valued } L^{1} \text {-Carathéodory function } \\
G_{1}:\left[t_{0}, \infty\right) \times \mathbb{R} \rightarrow[0, \infty) \text { with }|F(u, z)| \geq G_{1}(u, z) \\
\text { for }(u, z) \in\left[t_{0}, \infty\right) \times(0, \lambda),
\end{array}\right.
$$
$\left\{\begin{array}{l}\text { there exists a positive constant } M_{1} \text { such that if } 0<x \leq y \leq \lambda, \\ \text { then } G_{1}(t, x) \leq M_{1} G_{1}(t, y) \text { for } t \geq t_{0}\end{array}\right.$

and

$$
\int^{\infty} G_{1}(s, \mu \pi(s)) d s=\infty \text { for all } \mu>0
$$

hold, then $\lim _{t \rightarrow \infty} y(t) / \pi(t)=\infty$.

Proof. Choose $\mu>0$ so that

$$
\mu\left[1+\pi\left(t_{0}\right)\right] \leq \lambda
$$

and choose $T \geq t_{0}$ so that

$$
\int_{T}^{\infty} \frac{1}{a(s)} \int_{T}^{s} G(u, \lambda) d u d s \leq \frac{\mu}{2 M} .
$$

Let $E=B[T, \infty), Q=\{y \in B[T, \infty): \mu \pi(t) / 2 \leq y(t) \leq \lambda$ for $t \geq T\}$ and let $N: Q \rightarrow \mathscr{P}(E)$ be (here $y \in Q$ ),

$$
N(y)(t)=\frac{\mu}{2} \int_{t}^{\infty} \frac{d s}{a(s)}-\int_{t}^{\infty} \frac{1}{a(s)} \int_{T}^{s} F(u, y(u)) d u d s \text { for } t \geq T .
$$

It is easy to see from (2.4), (2.6), (2.15), (2.19) and (2.20) that

$$
N: Q \rightarrow C(Q)
$$

Similarly as in Theorem 2.1 one can also deduce that $N: Q \rightarrow C K(Q)$ is an upper semicontinuous, compact map. Theorem 1.1 guarantees that there exists $y \in Q$ with

$$
y(t) \in \frac{\mu}{2} \pi(t)-\int_{t}^{\infty} \frac{1}{a(s)} \int_{T}^{s} F(u, y(u)) d u d s \text { for } t \geq T .
$$

Also since $y \in Q$ we have $y(t) \in[\mu \pi(t) / 2, \lambda]$ for $t \geq T$, and $\lim _{t \rightarrow \infty} y(t)=0$ since

$$
|y(t)| \leq \frac{\mu}{2} \pi(t)+M \int_{t}^{\infty} \frac{1}{a(s)} \int_{T}^{s} G(u, \lambda) d u d s \rightarrow 0 \quad \text { as } t \rightarrow \infty .
$$


Next suppose (2.16)-(2.18) hold. We now show that

$$
\lim _{t \rightarrow \infty} \frac{y(t)}{\pi(t)}=\infty
$$

To see this notice there exists $\tau \in \mathscr{F}(y)$ with

Thus

$$
y(t)=\frac{\mu}{2} \pi(t)+\int_{t}^{\infty} \frac{1}{a(s)} \int_{T}^{s}[-\tau(u)] d u d s \quad \text { for } t \geq T .
$$

$$
\begin{aligned}
\lim _{t \rightarrow \infty} \frac{y(t)}{\pi(t)} & =\frac{\mu}{2}+\lim _{t \rightarrow \infty}\left[-\int_{T}^{t} \tau(u) d u\right] \\
& \geq \frac{\mu}{2}+\lim _{t \rightarrow \infty} \int_{T}^{t} G_{1}(u, y(u)) d u \\
& \geq \frac{\mu}{2}+\frac{1}{M_{1}} \lim _{t \rightarrow \infty} \int_{T}^{t} G_{1}\left(u, \frac{\mu}{2} \pi(u)\right) d u=\infty .
\end{aligned}
$$

REMARK 2.3. There is also an analogue of Theorem 2.2 if (2.4) is replaced by (2.13). We leave the details to the reader.

If we don't assume the condition $\pi\left(t_{0}\right)<\infty$, then it is also possible to obtain an analogue of Theorem 2.1 and Theorem 2.2. The idea here is to work with $C[T, \infty)$ instead of $B[T, \infty)$. For our next theorem, for notational purposes, let $R[t, x]=$ $\int_{x}^{t} d s / a(s)$ and $R(t)=R\left[t, t_{0}\right]$.

THEOREM 2.3. Suppose (2.2)-(2.4) and (2.6) hold and in addition assume the following conditions are satisfied

$$
\exists t_{1} \geq t_{0} \text { and } \lambda>0 \text { with } \int^{\infty} G\left(s, \lambda R\left[s, t_{1}\right]\right) d s<\infty
$$

and

$$
\left\{\begin{array}{l}
\text { there exists a positive constant } M \text { such that if } 0<x \leq \lambda \text {, then } \\
G\left(t, x R\left[t, t_{1}\right]\right) \leq M G\left(t, \lambda R\left[t, t_{1}\right]\right) \text { for } t \geq t_{1}
\end{array}\right.
$$

Then there is a nonoscillatory solution y of $\left(a(t) y^{\prime}(t)\right)^{\prime} \in F(t, y(t))$, a.e. $t \geq T$ with

$$
\lim _{t \rightarrow \infty} \frac{y(t)}{R(t)}=c_{0} \in(0, \lambda]
$$

here $T$ is chosen as in (2.24).

REMARK 2.4. (i) If $G$ is nondecreasing in the second variable (as in Remark2.1) then clearly (2.23) holds with $M=1$.

(ii) There is also an analogue of Theorem 2.3 if (2.13) replaces (2.4). 
Proof. Choose $T \geq t_{1}$ so that

$$
\int_{T}^{\infty} G\left(s, \lambda R\left[s, t_{1}\right]\right) d s \leq \frac{\lambda}{2 M} .
$$

Let $E=C[T, \omega)$,

$$
Q=\left\{y \in C[T, \infty): \frac{\lambda}{2} R[t, T] \leq y(t) \leq \lambda R\left[t, t_{1}\right] \text { for } t \geq T\right\}
$$

and let $N: Q \rightarrow \mathscr{P}(E)$ be (here $y \in Q$ ),

$$
N(y)(t)=\lambda \int_{T}^{t} \frac{d s}{a(s)}+\int_{T}^{t} \frac{1}{a(s)} \int_{T}^{s} F(u, y(u)) d u d s \quad \text { for } t \geq T .
$$

It is easy to see from (2.4), (2.6), (2.23) and (2.24) that

$$
N: Q \rightarrow C(Q) .
$$

A slight modification of the argument in [3, Theorem 1.1, page 1293] guarantees that $N: Q \rightarrow C K(Q)$ is an upper semicontinuous, compact map. Theorem 1.1 guarantees that there exists $y \in Q$ with

$$
y(t) \in \lambda \int_{T}^{t} \frac{d s}{a(s)}+\int_{T}^{t} \frac{1}{a(s)} \int_{T}^{s} F(u, y(u)) d u d s \quad \text { for } t \geq T .
$$

Now there exists $\tau \in \mathscr{F}(y)$ with

$$
y(t)=\int_{T}^{t} \frac{1}{a(s)}\left[\lambda+\int_{T}^{s} \tau(u) d u\right] d s \quad \text { for } t \geq T .
$$

There are two cases to consider, namely $\int^{\infty} d s / a(s)<\infty$ and $\int^{\infty} d s / a(s)=\infty$. If $\int^{\infty} d s / a(s)<\infty$ then

so

$$
0 \leq \int_{T}^{\infty} \frac{1}{a(s)}\left[\lambda+\int_{T}^{s} \tau(u) d u\right] d s \leq \lambda \int_{T}^{\infty} \frac{d s}{a(s)}<\infty,
$$

$$
\lim _{t \rightarrow \infty} \frac{y(t)}{R(t)}=\frac{\int_{T}^{\infty}\left[\lambda+\int_{T}^{s} \tau(u) d u\right] d s / a(s)}{\int_{t_{0}}^{\infty} d s / a(s)}=c_{0} .
$$

Notice that $c_{0} \in(0, \lambda]$ since

$$
\begin{aligned}
\int_{T}^{\infty} \frac{1}{a(s)}\left[\lambda+\int_{T}^{s} \tau(u) d u\right] d s & \geq \int_{T}^{\infty} \frac{1}{a(s)}\left[\lambda-M \frac{\lambda}{2 M}\right] d s \\
& =\frac{\lambda}{2} \int_{T}^{\infty} \frac{d s}{a(s)}
\end{aligned}
$$


and so

$$
\frac{(\lambda / 2) \int_{T}^{\infty} d s / a(s)}{\int_{t_{0}}^{\infty} d s / a(s)} \leq c_{0} \leq \frac{\lambda \int_{T}^{\infty} d s / a(s)}{\int_{t_{0}}^{\infty} d s / a(s)} \leq \lambda
$$

If $\int^{\infty} d s / a(s)=\infty$ then

$$
\int_{T}^{\infty} \frac{1}{a(s)}\left[\lambda+\int_{T}^{s} \tau(u) d u\right] d s \geq \frac{\lambda}{2} \int_{T}^{\infty} \frac{d s}{a(s)}=\infty
$$

so l'Hopital's rule implies

$$
\lim _{t \rightarrow \infty} \frac{y(t)}{R(t)}=\lambda+\int_{T}^{\infty} \tau(u) d u=c_{0}
$$

Notice that $\lambda / 2 \leq c_{0} \leq \lambda$ since $\lambda+\int_{T}^{\infty} \tau(u) d u \geq \lambda-M(\lambda / 2 M)=\lambda / 2$.

THEOREM 2.4. Suppose (2.2)-(2.4) and (2.6) hold and in addition assume the following conditions are satisfied

$$
\exists \lambda>0 \text { with } \int^{\infty} \frac{1}{a(s)} \int_{t_{0}}^{s} G(u, \lambda) d u d s<\infty
$$

and

$$
\left\{\begin{array}{l}
\text { there exists a positive constant } M \text { such that if } \lambda / 2 \leq x \leq \lambda, \text { then } \\
G(t, x) \leq M G(t, \lambda) \text { for } t \geq t_{0} .
\end{array}\right.
$$

Then there is a nonoscillatory solution y of $\left(a(t) y^{\prime}(t)\right)^{\prime} \in F(t, y(t))$, a.e. $t \geq T$ with $\lim _{t \rightarrow \infty} y(t)=c_{0} \in[\lambda / 2, \lambda]$. Here $T$ is chosen as in (2.28).

Proof. Choose $T \geq t_{0}$ with

$$
\int_{T}^{\infty} \frac{1}{a(s)} \int_{T}^{s} G(u, \lambda) d u d s \leq \int_{T}^{\infty} \frac{1}{a(s)} \int_{t_{0}}^{s} G(u, \lambda) d u d s \leq \frac{\lambda}{2 M}
$$

Let $E=C[T, \infty), Q=\{y \in C[T, \infty): \lambda / 2 \leq y(t) \leq \lambda$ for $t \geq T\}$ and let $N: Q \rightarrow \mathscr{P}(E)$ be (here $y \in Q$ ),

$$
N(y)(t)=\lambda+\int_{T}^{t} \frac{1}{a(s)} \int_{T}^{s} F(u, y(u)) d u d s \quad \text { for } t \geq T .
$$

It is easy to see from $(2.4),(2.6),(2.27)$ and $(2.28)$ that

$$
N: Q \rightarrow C(Q)
$$


Also (as in Theorem 2.3) we have that $N: Q \rightarrow C K(Q)$ is an upper semicontinuous, compact map. Theorem 1.1 guarantees that there exists $y \in Q$ with

$$
y(t) \in \lambda+\int_{T}^{t} \frac{1}{a(s)} \int_{T}^{s} F(u, y(u)) d u d s \text { for } t \geq T .
$$

Notice that $y(t) \in[\lambda / 2, \lambda]$ for $t \geq T$. Now there exists $\tau \in \mathscr{F}(y)$ with

$$
y(t)=\lambda+\int_{T}^{t} \frac{1}{a(s)} \int_{T}^{s} \tau(u) d u d s \quad \text { for } t \geq T .
$$

Since $y$ is monotonic, $\lim _{t \rightarrow \infty} y(t)$ exists and

$$
\lim _{t \rightarrow \infty} y(t)=\lambda+\int_{T}^{\infty} \frac{1}{a(s)} \int_{T}^{s} \tau(u) d u d s .
$$

REMARK 2.5. (i) There is an analogue of Theorem 2.4 if (2.13) replaces (2.4).

(ii) In Theorem 2.4, it is possible to replace (2.6), (2.26) and (2.27) with

$$
\exists \lambda>0 \text { with } \int^{\infty} \frac{1}{a(s)} \int_{I_{0}}^{s} \sup _{w \in[\lambda / 2, \lambda]}|F(u, w)| d u d s<\infty,
$$

and the result is again true. The proof only involves a slight modification of the above argument.

THEOREM 2.5. Suppose (2.2)-(2.4) and (2.6) hold and in addition assume the following conditions are satisfied

$$
\exists \lambda>0, \mu>0 \text { with } \int^{\infty} G\left(s, \mu+\lambda \int_{t_{0}}^{s} \frac{d x}{a(x)}\right) d s<\infty
$$

and

$$
\text { for } 0<x \leq y \text { we have } G(t, x) \leq G(t, y) \text { for } t \geq t_{0} \text {. }
$$

Then there is a nonoscillatory solution y of $\left(a(t) y^{\prime}(t)\right)^{\prime} \in F(t, y(t))$, a.e. $t \geq T$. Here $T$ is chosen as in (2.34).

If, in addition,

$$
\int_{T}^{\infty} \frac{1}{a(s)} \int_{s}^{\infty} G\left(u, \mu+\lambda \int_{t_{0}}^{u} \frac{d x}{a(x)}\right) d u d s=\infty,
$$

then $\lim _{t \rightarrow \infty} y(t)=\infty$.

Proof. Choose $T \geq t_{0}$ so that

$$
\int_{T}^{\infty} G\left(s, \mu+\lambda \int_{t_{0}}^{s} \frac{d x}{a(x)}\right) d s \leq \lambda .
$$


Let $E=C[T, \infty)$,

$$
Q=\left\{y \in C[T, \infty): \mu \leq y(t) \leq \mu+\lambda \int_{t_{0}}^{t} \frac{d x}{a(x)} \text { for } t \geq T\right\}
$$

and let $N: Q \rightarrow \mathscr{P}(E)$ be (here $y \in Q$ ),

$$
N(y)(t)=\mu+\int_{T}^{t} \frac{1}{a(s)}\left[\lambda+\int_{T}^{s} F(u, y(u)) d u\right] d s \quad \text { for } t \geq T .
$$

It is easy to see from (2.4), (2.6), (2.32) and (2.34) that

$$
N: Q \rightarrow C(Q) .
$$

Also (as in Theorem 2.3) we have that $N: Q \rightarrow C K(Q)$ is an upper semicontinuous, compact map. Theorem 1.1 guarantees that there exists $y \in Q$ with

$$
y(t) \in \mu+\int_{T}^{t} \frac{1}{a(s)}\left[\lambda+\int_{T}^{s} F(u, y(u)) d u\right] d s \text { for } t \geq T .
$$

Now there exists $\tau \in \mathscr{F}(y)$ with

$$
y(t)=\mu+\int_{T}^{t} \frac{1}{a(s)}\left[\lambda+\int_{T}^{s} \tau(u) d u\right] d s \text { for } t \geq T .
$$

Suppose (2.33) holds. Then

$$
\begin{aligned}
y(t) \geq & \mu+\int_{T}^{t} \frac{1}{a(s)}\left[\int_{T}^{\infty} G\left(u, \mu+\lambda \int_{t_{0}}^{u} \frac{d x}{a(x)}\right) d u+\int_{T}^{s} \tau(u) d u\right] d s \\
\geq & \mu+\int_{T}^{t} \frac{1}{a(s)}\left[\int_{T}^{\infty} G\left(u, \mu+\lambda \int_{t_{0}}^{u} \frac{d x}{a(x)}\right) d u\right. \\
& \left.-\int_{T}^{s} G(u, y(u)) d u\right] d s \\
\geq & \mu+\int_{T}^{t} \frac{1}{a(s)}\left[\int_{T}^{\infty} G\left(u, \mu+\lambda \int_{t_{0}}^{u} \frac{d x}{a(x)}\right) d u\right. \\
& \left.\left.\left.-\int_{T}^{s} G\left(u, \mu+\lambda \int_{t_{0}}^{u} \frac{d x}{a(x)}\right) d u\right]_{d s}(u) \int_{t_{0}}^{u} \frac{d x}{a(x)}\right) d u\right] d s \stackrel{1 \rightarrow \infty}{\longrightarrow} \infty . \\
= & \mu+\int_{T}^{t} \frac{1}{a(s)}\left[\int_{s}^{\infty} G(u, \mu+\lambda .\right.
\end{aligned}
$$

REMARK 2.6. (i) There is an analogue of Theorem 2.5 if (2.13) replaces (2.4).

(ii) Notice that if $\int_{T}^{\infty}\left(\int_{s}^{\infty} G(u, \mu) d u\right) d s / a(s)=\infty$, then (2.33) holds since (2.32) guarantees that $G\left(s, \mu+\lambda \int_{1_{0}}^{s} d x / a(x)\right) \geq G(s, \mu)$ for $s \geq T$. 


\section{References}

[1] R. P. Agarwal, S. R. Grace and D. O'Regan, 'On nonoscillatory solutions of differential inclusions', Proc. Amer. Math. Soc. 131 (2003), 129-140.

[2] R. P. Agarwal, M. Meehan and D. O'Regan, Fixed point theory and applications (Cambridge University Press, Cambridge, 2001).

[3] R. P. Agarwal and D. O'Regan, 'Nonlinear operator inclusions on the half line', Math. Comput. Modelling 32 (2000), 1287-1296.

[4] M. Cecchi, M. Marini and P. Zecca, 'Existence of bounded solutions for multivalued differential systems', Nonlinear Anal. 9 (1985), 775-786.

[5] N. Dunford and J. Schwartz, Linear operators I. General theory (Interscience, New York, 1958).

[6] L. H. Erbe, Q. K. Kong and B. G. Zhang, Oscillation theory for functional differential equations (Marcel Dekker, New York, 1995).

[7] W. Jingfa, 'On second order quasilinear oscillations', Funkcial. Ekvac. 41 (1998), 25-54.

[8] T. Kusano, A. Ogata and H. Usami, 'Oscillation theory for a class of second order quasilinear ordinary differential equations with applications to partial differential equations', Japan J. Math. 19 (1993), 131-147.

[9] G. S. Ladde, V. Lakshmikantham and B. G. Zhang, Oscillation theory of differential equations with deviating arguments (Marcel Dekker, New York, 1987).

[10] B. Singh, 'Asymptotic nature of nonoscillatory solutions of $n$th order retarded differential equations', SIAM J. Math. Anal. 6 (1975), 784-795.

Department of Mathematical Sciences

Florida Institute of Technology

Melbourne FL 32901

USA

e-mail: agarwal@fit.edu

\section{Department of Mathematics}

National University of Ireland

Galway

Ireland
Department of Engineering Mathematics

Cairo University

Orman, Giza 12221

Egypt 